\title{
Absence of relational determination in the rod-and-frame effect
}

\author{
SHELDON M. EBENHOLTZ \\ University of Wisconsin, Madison, Wisconsin
}

\begin{abstract}
Relational features between a rod and frame that might facilitate an angle-detection process were manipulated in two ways: first, by varying rod lengths with retinal eccentricities of $3.8^{\circ}$, $7.5^{\circ}$, and $14.8^{\circ}$ in a frame with inner dimensions of $35.3^{\circ} \times 35.3^{\circ}$, and second, by eliminating the sides, leaving only the corners of the frame. The converse case, in which corners were eliminated but sides left intact, represented the control condition. In neither case did the opportunity to use relational features enhance the rod-and-frame effect (RFE). In a frame with inner dimensions of $52.5^{\circ} \times 52.5^{\circ}$, when line length had an eccentricity of about $25^{\circ}$, reducing it to $3^{\circ}$ did significantly reduce the RFE, but only for those who scored in the upper $50 \%$ as measured on the full-frame RFE. An explanation is proposed based upon visual-vestibular interactions between receptive fields in peripheral retina and orientation detectors in the vestibular nucleus.
\end{abstract}

The rod-and-frame effect (RFE) represents the influence of a large tilted surrounding frame on the apparent orientation of a line included within it (Witkin \& Asch, 1948). It is conventionally viewed as an instance of relational determination in perception (Koffka, 1935), according to which the apparent orientation of the line is largely predicated on the specific angle the line makes relative to a frame edge. Thus, to take an extreme case, if the frame were to determine completely the apparent orientation of the line, the latter would appear upright when it either was parallel with the laterally oriented frame edges or made a $90^{\circ}$ angle with the top and bottom edges of the tilted frame. In either case, it is obvious that judgments of angle are critical to this process, and, indeed, systematic angle illusions of over- and underestimation have been shown to exist in rod-and-frame displays (Goodenough, Oltman, Sigman, Rosso, \& Mertz, 1979).

The relational approach also is consistent with a cognitive problem-solving view of the RFE (DiLorenzo \& Rock, 1982) according to which the line setting is governed by the extent of perceived frame tilt. In this view, the line would appear upright when set at an angle, with respect to, for example, the most nearly parallel frame edge, that was equal in magnitude but opposite in direction to the apparent tilt of the frame. Since, as DiLorenzo and Rock have shown for frames of relatively large visual angle, frame tilt tends to be underestimated, the angle between line and frame would be set too low. The net result would be a line tilted (relative to gravitational vertical) in the direction of the frame by an amount equal to the angular difference between actual and apparent frame tilt.

This material is based upon work supported by the National Science Foundation under Grant BNS 8201411. Thanks are expressed to John W. Utrie, Jr., for his aid in directing the experiments.

The author's mailing address is: Department of Psychology, University of Wisconsin, Madison, WI 53706
Given the importance of detecting the angular relation of line to frame in the problem-solving approach, and the great significance of relational determination in Gestalt theory, we decided to investigate relational factors in the rod-and-frame effect by manipulating the ease by which angle detection might be assumed to occur. This was accomplished in two ways, the first of which was to vary line length and hence spatial separation between the ends of the line and the frame edge. With increasing separation and decreasing line length, the salience of the angular relationship may be expected to diminish (Andrews, 1967; Sulzer \& Zener, 1953) ${ }^{1}$ and, as a consequence, line setting should become progressively more independent of the frame orientation.

In the second approach, approximately $80 \%$ of each side of the frame was eliminated, leaving only four corners. ${ }^{2}$ If the absence of frame edges represents the removal of relational features from the display, then, just as in the case of a short line, angle detection should be made more difficult and the RFE should diminish.

\section{EXPERIMENTS 1 AND 2}

\section{Method and Design}

Twenty-four volunteer subjects, evenly divided among male and female, took part in Experiment 1. The subjects viewed a square luminous frame, $35.6 \mathrm{~cm} \mathrm{side} \mathrm{e}^{-1}$ at a distance of $53.5 \mathrm{~cm}$, with a $22^{\circ}$ clockwise tilt. The task was to adjust a luminous line, rotated about an axis at the frame center, to a position corresponding to the forehead-chin or 6 to 12 o'clock direction. In a repeatedmeasures design, line lengths of $7.1,14.1$, and $28.3 \mathrm{~cm}$, all with a $.2-\mathrm{cm}$ width, were used in counterbalanced order over the 24 subjects.

In terms of retinal angle, the distance between the inner edges of opposite sides of the frame was $35.3^{\circ}$ and the short, medium, and long lines projected $7.6^{\circ}, 15.0^{\circ}$, and $29.6^{\circ}$, respectively. With the line parallel to one of the frame sides, these corresponded to a gap size, between line tip and frame edge, of $14.3^{\circ}, 10.6^{\circ}$, and $3.1^{\circ}$. Two adjustments of the line to apparent egocentric vertical 
were made, one from each starting position at $25^{\circ}$ clockwise and counterclockwise of true vertical. For each line length, settings were made first without the frame and then again with the frame present. The algebraic difference between the mean settings with and without the frame constituted the rod-and-frame effect (RFE).

In Experiment 1, the subjects were cautioned to direct their attention to the center of the line while it was being rotated into the apparently vertical position. They were then directed to look the line over to ensure that it occupied the desired location; bracketing was then permitted. In Experiment 2, conditions were identical except that the instruction to attend to the center of the line during the movement was omitted. Only the short and long lines were used and eight additional subjects, four male and four female, took part.

\section{Results}

The results, shown in Table 1 , indicate that despite the fourfold shift in line length, in neither experiment was the RFE significantly influenced by this variable [in Experiment 1, $F(2,46)=.19, p>.05$; in Experiment 2, $\mathfrak{t}(7)=.33, p>.05]$. Null effects also occurred in the analysis of the upper and lower terciles of RFE scores as judged by performance on the long-line condition of Experiment 1.

Further analyses of the data of Experiment 1 all failed to provide evidence for an influence of line length. These included the line settings made in the absence of the frame $[F(2,46)=2.42, \mathrm{p}>.05]$, which were consistent with the null results reported by Wade (1969) for lines $2.1 \mathrm{~cm}$ wide and either 15.5 or $91.5 \mathrm{~cm}$ long $\left(4.9^{\circ}\right.$ and $28.5^{\circ}$, respectively). Likewise, the absolute value of the range in line settings between each of the two starting positions failed to achieve significance. Frame presence or absence, $[F(1,23)=.095, p>.05]$ and line length $[F(2,46)=$ $1.30, p>.05]$ produced no significant effects. The interaction of the two also was not significant $[F(2,46)=$ $.114, \mathrm{p}>.05]$.

The shortest line used projected a retinal angle $7.6^{\circ}$ and provided a gap between line and frame edge of about $14.3^{\circ}$. Yet, no influence on line settings was discernible. At least for the present range of sizes, it is clear that the RFE is not sensitive to a coplanar line-frame adjacency effect (Gogel, 1978). This result is consistent with the absence of a depth-adjacency effect in large frames (Ebenholtz \& Glazer, 1982).

Experiment 3 attempted to replicate the results of Experiments 1 and 2 with a frame and long line that extended considerably further into the periphery than the previous displays. In addition, a second approach to the manipulation of relational features was explored. The underlying reasoning was to the effect that removal of the sides

Table 1

Rod-and-Frame Effect (Degrees) as a Function of Line Length

\begin{tabular}{|c|c|c|c|c|c|c|}
\hline & \multicolumn{6}{|c|}{ Line Length $(\mathrm{cm})$} \\
\hline & \multicolumn{2}{|c|}{ Short (7.1) } & \multicolumn{2}{|c|}{ Medium (14.1) } & \multicolumn{2}{|c|}{ Long (28.3) } \\
\hline & $\mathbf{M}$ & $\sigma_{M}$ & $\mathbf{M}$ & $\sigma_{M}$ & $\mathbf{M}$ & $\sigma_{\mathrm{M}}$ \\
\hline Experiment 1 & 4.67 & .81 & 4.44 & .80 & $4 . \overline{57}$ & .76 \\
\hline Upper Tercile & 7.88 & 1.03 & 8.09 & 1.17 & 8.32 & .98 \\
\hline Lower Tercile & 0.59 & .67 & 0.64 & .86 & 0.54 & .53 \\
\hline Experiment 2 & 4.01 & 1.28 & & & 4.23 & 1.61 \\
\hline
\end{tabular}

Table 2

Rod-and-Frame Effect (Degrees) by Line Length (Centimeters) and With the Long Line in Sides-Only and Corners-Only Displays

\begin{tabular}{|c|c|c|c|c|c|c|c|c|}
\hline & \multicolumn{4}{|c|}{ Line Length } & \multicolumn{4}{|c|}{ Display Type } \\
\hline & \multicolumn{2}{|c|}{ Long (95.5) } & \multicolumn{2}{|c|}{ Short (10.8) } & \multicolumn{2}{|c|}{ Sides } & \multicolumn{2}{|c|}{ Corners } \\
\hline & $\mathbf{M}$ & $\sigma_{\mathbf{M}}$ & $\mathbf{M}$ & $\sigma_{M}$ & $\mathrm{M}$ & $\sigma_{\mathrm{M}}$ & $\mathbf{M}$ & $\sigma_{M}$ \\
\hline Entire Group & 4.72 & .63 & 3.28 & .39 & 2.94 & .51 & 2.52 & .55 \\
\hline Eight Highest Scores & 6.97 & .37 & 4.28 & .39 & 4.19 & .69 & 4.00 & .56 \\
\hline Eight Lowest Scores & 2.47 & .37 & 2.28 & .46 & 1.69 & .43 & 1.03 & .61 \\
\hline
\end{tabular}

of a frame should serve to eliminate relational features otherwise present in the rod-and-frame display. The presence of sides, on the other hand, should readily permit an angle detection process to operate by mediating the use of relational features in the display.

\section{EXPERIMENT 3}

\section{Method and Design}

Sixteen subjects, evenly divided among male and female gender, took part in two sessions, approximately 1 week apart. In the first session, the RFE was measured with a full frame, $106.5 \times 2.4 \mathrm{~cm}$ side $^{-1}$, at a distance of $103.2 \mathrm{~cm}$. The subjects were exposed in succession to two displays, each containing either a long line of $95.5 \times 1 \mathrm{~cm}$ or a short line of $10.8 \times 1 \mathrm{~cm}$ in counterbalanced order. In degrees of arc at the retina, one side of the frame projected $54.6^{\circ}$, the long and short lines equaling $49.7^{\circ}$ and $6.0^{\circ}$, respectively.

In the second session, the subjects again took part in two conditions in counterbalanced order. In Condition $\mathrm{C}$ (corners), portions of the frame were eliminated to the extent of $79.5 \%$ of each side, leaving only corners with an outer edge of $10.9 \times 10.9 \mathrm{~cm}$. In Condition $S$ (sides), corners were eliminated from view and only sides of length $84.7 \mathrm{~cm}$ remained visible. The long line of the first session was used. Other procedures were as previously described.

\section{Results}

The results of both sessions are represented in Table 2 . It is interesting to note that shortening the line length significantly reduced the RFE overall [ $\mathrm{t}(15)=2.80, \mathrm{p}<$ $.01]$ and for the eight high scorers $[t(7)=4.01, p<.01]$, but not for the eight low scorers $[t(7)=0.39, p>.05]$. Thus, high scorers are sensitive to variations in line length when a rod and frame with a relatively large retinal angle are used.

The data of the second session showed that the two conditions produced equivalent RFEs, since in no case did the sides vs. corners comparison prove significant. In all cases, however, these conditions led to a significant decrement $(\mathrm{p}<.01)$ in the RFE relative to the corresponding full-frame condition [ts $(15)=4.52$ and 5.00 for comparisons with Conditions $S$ and $C$, respectively]. Thus, the partial frames reduced the RFE, but the presence or $a b-$ sence of the sides did not differentiate between these conditions.

\section{DISCUSSION}

The failure of the RFE to be sensitive to as much as a fourfold reduction in line length in Experiments 1 and 
2 lends doubt to any characterization of the rod-and-frame task as an instance of relational determination. Likewise, the cognitive, or problem-solving, view of the RFE as developed by DiLorenzo and Rock (1982, p. 544), insofar as it rests upon the detection of the angular relation of the rod to the frame, was not supported. Such a process should have been sensitive to the large changes in contiguity presently examined. Of course, it is possible mentally to extend the line to the frame edge and thereby account for the absence of line-length effects. This, however, is inconsistent with retrospective reports by most subjects to the effect that they tend to ignore the frame and to concentrate on the line orientation.

Positing a mental extension process also is inconsistent with the decrement found in the short-line-length condition of Experiment 3, and hence some other explanation for the pattern of results is required.

The second manipulation of relational features also failed to support an angle-estimation process. The results showed equivalence in performance on the RFE between a corner display, without sides, where direct angular relations between line and frame were absent, and a "sides without corners" display that permitted such relationships to be used. From this finding, it would be dubious to argue that the absence of opportunity to use angular relationships in the large-frame/short-line condition was responsible for its effect. ${ }^{3}$

It is conceivable that a cognitive angle-estimation strategy would be more frequently encountered when target orientation was adjusted according to gravitational instructions (Ebenholtz \& Callan, 1980; Sigman, Goodenough, \& Flannagan, 1979) as opposed to the egocentric instructions of the present study. By referring the subject to the gravitationally defined direction, compensation for apparent body tilt may be encouraged, along with additional cognitive strategies which may include judgments of the relative angle between line and frame edge. It remains to be determined whether evidence for such an angle-detection process would become more likely with the change from an egocentric to a gravitational reference system.

On the basis of presently available evidence, we propose that the rod-and-frame effect on egocentric orientation does not depend on the apparent angular relationship between rod and frame, the purpose of the rod being essentially to provide a convenient method of measurement. Rather, the effect can be understood as completely determined by the automatic (i.e., not cognitively mediated) influence of the peripheral representation of the frame on the egocentric orientation-coordinate system (Ebenholtz \& Glaser, 1982; Ebenholtz \& Utrie, 1983).

The results of both sessions of Experiment 3 are consistent with a neurophysiological model of visualvestibular interactions (Dichgans \& Brandt, 1978; Waespe \& Henn, 1977). In this approach, peripheral receptive fields are assumed to process the image of the tilted frame patterns. Populations of static orientation detectors, appropriate to the particular frame tilt, will in turn be stimu- lated, probably at the vestibular nucleus (Daunton \& Thomsen, 1979), to initiate the chain of events ultimately producing the RFE. Since the effect is assumed to be mediated by populations of orientation detectors, the magnitude of the effect may be thought to be modulated by alterations in their numbers. In this way, we may account for the decrease in RFE between full- and partial-frame conditions. ${ }^{4}$ Similarly, the long line in Experiment 3 may have been particularly useful in contributing to the stimulation of receptive fields in the retinal periphery along with that provided by the peripherally imaged frame.

Assume, for example, that the frame induces an effect of some magnitude, so that the line must be rotated in the direction of the frame. Because of its great length, however, the line thereby introduces into the periphery yet additional tilted contours, which then contribute still further to the overall effect. Thus, once the tilted frame had its effect by causing the line to be slightly rotated in order to appear upright, the tilted peripheral image of the line itself may have contributed still further, in the form of a positive feedback loop. Failure to find this effect in Experiments 1 and 2 may simply reflect the fact that the lines were not sufficiently peripheral to add significantly to the RFE beyond the contribution of the frame.

The equivalent effects of corner and side conditions probably represent a balance between two factors, both of which are capable of modulating the RFE. These are pattern eccentricity (Dichgans \& Brandt, 1978; Ebenholtz, 1977) and numbers of orientation detectors implicated. Although the corners represented only $23.2 \%$ of the linear extent of the sides, they were represented at sites considerably more peripheral than the sides. For example, the distance between the inner edges of the sides projected a retinal angle of $52.5^{\circ}$ for a retinal eccentricity of about $26^{\circ}$. On the other hand, the diagonal separation between corners projected $72.2^{\circ}$ for an eccentricity of $36^{\circ}$. Thus, the obtained equivalence may simply have resulted from a fortuitous balance between these two factors.

\section{REFERENCES}

ANdrews, D. P. (1967). Perception of contour orientation in the central fovea. Part U. Spatial integration. Vision Research, 7, 999-1013.

Cross, H. A. (1973). Judgment of rod verticality as a function of subject classification and frame pattern. Bulletin of the Psychonomic Society, 2, 23-24.

DAunton, N., \& Thomsen, D. (1979). Visual modulation of otolith units in cat vestibular nuclei. Experimental Brain Research, 37, 173-176.

DichGans, J., \& BrandT, T. (1978). Visual-vestibular interactions: Effects on self-motion perception and postural control. In R. Held, $H$. Leibowitz, \& H.-L. Teuber (Eds.), Handbook of sensory physiology: Vol. VIII. Perception. New York: Springer.

DiLorenzo, J. R., \& Rock, I. (1982). The rod-and-frame effect as a function of the righting of the frame. Journal of Experimental Psychology: Human Perception and Performance, 8, 536-546.

EBENholtz, S. M. (1977). Determinants of the rod and frame effect: The role of retinal size. Perception \& Psychophysics, 22, 537-538.

Ebenholtz, S. M., \& Callan, J. W. (1980). Modulation of the rod and frame effect: Retinal angle vs. apparent size. Psychological Research, 42, 327-334.

EBenholtz, S. M., Glaser, G. W. (1982). Absence of depth process- 
ing in the large-frame rod-and-frame effect. Perception \& Psychophysics, 32, 134-140.

EbenholtZ, S. M., \& UTRIE, J. W., JR. (1983). Peripheral circular contours inhibit the visual orientation control system. Aviation Space and Environmental Medicine, 54, 343-346.

GoGEL, W. C. (1978). The adjacency principle in visual perception. Scientific American, 238, 126-139.

Goodenough, D. R., Oltman, P. K., Sigman, E., Rosso, J., \& MerTZ, H. (1979). Orientation contrast effects in the rod-and-frame test. Perception \& Psychophysics, 25, 419-424.

KoffKA, K. (1935). Principles of gestalt psychology. New York: Harcourt, Brace.

Sigman, E., Goodenough, D. R., \& Flannagan, M. (1979). Instructions, illusory self-tilt and the rod-and-frame test. Quarterly Journal of Experimental Psychology, 31, 155-165.

Sulzer, R. L., \& Zener, K. E. (1953). A quantitative analysis of relations between stimulus determinants and sensitivity of the visual perception of parallelness. American Psychologist, 8, 444.

WADE, N. J. (1969). The effect of stimulus line variations on visual orientation with head upright and tilted. Australian Journal of Psychology, 21, 177-185.

WAESPE, W., HENN, V. (1977). Neuronal activity in the vestibular nuclei of the alert monkey during vestibular and optokinetic stimulation. Experimental Brain Research, 27, 523-538.

WITKIN, H. A., \& AsCH, S. E. (1948). Studies in space orientation: IV. Further experiments on perception of the upright with displaced visual fields. Journal of Experimental Psychology, 38, 762-782.

\section{NOTES}

1. Andrews (1967) showed an increase in precision in settings to parallel with increasing line length up to about $9^{\prime}$ arc. Sulzer and Zener (1953) obtained an increase in variance in settings to parallel with increasing spacing between lines (from $.06^{\circ}$ to $7.63^{\circ}$ ) and with decreasing line length, the latter varying from $.24^{\circ}$ to $3.81^{\circ}$.

2. Cross (1973) reported a signal detection study of performance on a rod-and-frame task in which comparisons were made among a full frame, a frame with corners only, and a frame with sides only. No measures of the magnitude of RFE, however, were given.

3. One reviewer has suggested that, for undetermined reasons, eliminating sides or corners might reduce the righting effect. This would not, however, reduce the need, in the cognitive theory, for an angle estimation process. It is the sensitivity of the latter to the manipulation of line-edge contiguity and the sheer presence or absence of the frameedge that has been presumed in the present study. These manipulations also represent operational definitions of relational factors, although they are not by any means exhaustive.

4. Although low scorers showed significant RFEs in the two line-length conditions, they did not differ significantly from each other. The absence of effect of line length among low scorers in Experiment 3 follows from the premise that such individuals are less influenced by images of tilted linear elements in peripheral retina than are high scorers. This suggests that low and high scorers may differ in peripheral spatial frequency sensitivities.

(Manuscript received February 21, 1984; revision accepted for publication February 26, 1985.) 